\title{
Time-dependent persistence of enhanced immune response by a potential probiotic strain Lactobacillus paracasei subsp. paracasei NTU 101
}

\author{
Yueh-Ting Tsai ${ }^{\mathrm{a}}$, Po-Ching Cheng ${ }^{\mathrm{b}}$, Chia-Kwung Fan ${ }^{\mathrm{c}}$, Tzu-Ming Pan ${ }^{\mathrm{a}, *}$ \\ a Institute of Microbiology and Biochemistry, College of Life Science, National Taiwan University, Taipei 10617, Taiwan \\ b Institute of Tropic Medicine, National Yang-Ming University, Taipei 11221, Taiwan \\ c Department of Parasitology, College of Medicine, Taipei Medical University, Taipei 11031, Taiwan
}

\section{A R T I C L E I N F O}

\section{Article history:}

Received 27 February 2008

Received in revised form 10 July 2008

Accepted 19 August 2008

\section{Keywords:}

Lactic acid bacteria

Lactobacillus paracasei subsp. paracasei NTU

101

Innate immune response

Immunomodulation

\begin{abstract}
A B S T R A C T
The possible time-dependent role of lactic acid bacteria (LAB) in immunomodulation was investigated in BALB/c mice fed daily with Lactobacillus paracasei subsp. paracasei NTU 101 ( $10^{8}$ colony forming units) for 3 , 6, and 9 weeks, and following feeding with Lactobacillus-free food for a further 7 days. We observed upregulation of the antigen-presenting ability of dendritic cells, and expression of natural killer group-2 D (NKG2D) molecules capable of trigger natural killer cell-mediated cytotoxicity. Lymphocyte proliferation and antibody production were also significantly increased in mice after treatment. Innate and adaptive immunity remained constant even at the most protracted feeding time, indicative of the time dependence of the bacterial-mediated enhanced immunity. To better correlate intestinal microflora with immunity, the intestinal contents of probiotics and harmful microorganisms were determined. Results showed an altered intestinal microflora, with increases in bifidobacteria and lactobacilli and a decreased content of Clostridium perfringens after feeding with $L$. paracasei subsp. paracasei NTU 101. It is possible that persistent activation of immunity might be induced by intestinal probiotics.
\end{abstract}

(c) 2008 Elsevier B.V. All rights reserved.

\section{Introduction}

Lactic acid bacteria (LAB) are a major component of the commensal microbial flora of the human gastrointestinal tract and are frequently used as probiotics for fermentation of food products. Dietary supplementation with live beneficial bacteria promotes health and reduces the risk of various diseases (Ahrne et al., 1998). Lactobacilli play important roles in enhancement of immunity, maintenance of intestinal microbial balance and prevention of gastrointestinal infection. Immunomodulation is a very important factor contributing to anti-inflammatory, antiinfection and anti-tumor effects. It has been proposed that lactobacilli can be used for immune-stimulation to increase early lines of defense against invading pathogens (Goldin, 1998).

However, the mechanisms of immunomodulation are still unknown. It has been demonstrated that the cell wall of Lactobacillus contains immunomodulatory components such as polysaccharide, peptidoglycan and bacteriocin, which may be influential in activating immune responses (Meydani and Ha, 2000; Cotter et al., 2005). In murine models, several lactobacilli strains enhance both innate and adaptive immune response through the induction of dendritic cell (DC) maturation and further stimulate immune cells to release pro-inflammatory cytokines including

\footnotetext{
* Corresponding author. Institute of Microbiology and Biochemistry, College of Life Science, National Taiwan University, Taipei, Taiwan, No. 1, Sec. 4, Roosevelt Road, Taipei, 10617, Taiwan. Tel.: +886 2 33664519x10; fax: +886223627044.

E-mail address: tmpan@ntu.edu.tw (T.-M. Pan).
}

tumor necrosis factor-alpha (TNF- $\alpha$ ), interferon-gamma (IFN- $\gamma$ ) and interleukin-12 (IL-12) (Perdigon et al., 1999). Previous studies demonstrated that different lactobacilli strain and dosage may cause different immune response (Gill et al., 2000; Maassen et al., 2000), but the correlation between immunomodulorty and lactobacilli remains unclear.

The innate immune response serves not only as the first line of defense but also has a crucial role in the development of subsequent adaptive immune responses. Mechanisms of innate immunity include enhancement of antigen presentation, phagocytosis on antigen presenting cells (APCs) and cytotoxicity by natural killer (NK) cells, which can all lead to killing of transformed cells in a seemingly nonspecific fashion (Aderem and Underhill, 1999). Macrophages phagocytose the infected cells and then present antigens (Ags) to T and B lymphocytes, preluding the production of antibodies, cytokines and chemokines to modulate immune responses (Morrissette et al., 1999). Recent studies have shown in vitro exposure of bone marrow-derived DC to Lactobacillus can up-regulate surface MHC class II, B7-1 (CD80) and B7-2 (CD86) on DCs, and skew T cells from T helper 2 (Th2) toward Th1 responses, promoting humoral and cell mediated immunity (Mohamadzadeh et al., 2005). NK cells are specialized lymphocytes of the innate immune system capable of attacking virus-infected cells or transformed tumor cells via NKG2D triggered NK cell-mediated cytotoxicity (Diefenbach et al., 2000). In a previous study, Lactobacillus casei strain Shirota (LCS) increased NK cell activity was observed in mice and humans after ingestion of fermented milk (Hori et al., 2003; Takeda and Okumura, 2007). 
In our previous studies, Lactobacillus paracasei subsp. paracasei NTU 101 was isolated from human infant feces. Isolates display the hallmark features of good survival at low $\mathrm{pH}$, tolerance to high bile concentration (Pan et al., 2002). Furthermore, the L. paracasei subsp. paracasei NTU 101-fermented milk could change of lactic acid production, sensory characteristics and ability to reduce serum cholesterol in hypercholesterolemic rats (Lin et al., 2004; Chiu et al., 2006). We have demonstrated that supplementation of human diets with $L$. paracasei subsp. paracasei NTU 101 may help for health benefit, but the effects of Lactobacillus on immune response were remain unclear. Therefore, the objective of the present study was to evaluate the mechanism of enhancing immune response by applying Lactobacillus for differing times using a murine model. The results demonstrate that supplementation of the diet with $L$. paracasei subsp. paracasei NTU 101 can persistently enhance antigen presenting ability, cytotoxicity activity, lymphocyte proliferation and antibody production, even following termination of bacteria-supplemented diet. Furthermore, the persistence activities can be induced by the increase of intestinal probiotics.

\section{Materials and methods}

\subsection{Bacteria and growth conditions}

L. paracasei subsp. paracasei NTU 101 was inoculated in MRS broth (BD Biosciences, San Jose, CA, USA) and grown under anaerobic conditions using an atmosphere generation system (Oxoid, Basingstoke, Hampshire, England) at $37^{\circ} \mathrm{C}$ for $48 \mathrm{~h}$. Thereafter, bacteria were resuspended in MRS broth to a final concentration of $1 \times 10^{9} \mathrm{CFU}$ (colony-forming unit)/mL. The number and viability of the lactobacilli were determined by anaerobic cultivation on MRS plates (BD Biosciences) for $48 \mathrm{~h}$.

\subsection{Experimental animals}

Specific-pathogen-free (SPF), male, 6-8 weeks old BALB/c mice or Sprague-Dawley rats were obtained from the National Laboratory Animal Center (Taipei, Taiwan). Animal experiments were conducted in accordance with regulations in the NIH Guide for the Care and Use of Laboratory Animals (DHHS publication No. NIH 85-23, revised 1996). Animals were provided with water and Labdiet 5001 chow (PMI Nutrition International, St. Louis, MO, USA) ad libitum. Animals were housed in a plastic cage and kept under pathogen-free conditions at $22 \pm 1{ }^{\circ} \mathrm{C}$ and humidity of $55 \pm 2 \%$ with a $12 \mathrm{~h}$ alternating light-dark cycle.

\subsection{Evaluation of immune response on splenocytes}

Two experiments were performed. In the first experiment, BALB/c mice were divided into seven groups. All groups received $10^{9} \mathrm{CFU} / \mathrm{mL}$ L. paracasei subsp. paracasei NTU 101 in $0.1 \mathrm{~mL}$ MRS broth by oral administration once a day. Four groups received the bacteria for $0,3,6$ and 9 consecutive weeks. The remaining three groups were fed for 3, 6 and 9 weeks, prior to feeding with bacteria-free chow for 7 days (3W-7d, $6 \mathrm{~W}-7 \mathrm{~d}$ and $9 \mathrm{~W}-7 \mathrm{~d}$, respectively). Control groups received the same volume of sterile MRS broth at the same times. All data were from three independent experiments. After the final treatment, the mice were sacrificed, the spleen was removed and placed in a Petri dish, and then washed to flush out the leucocytes. The leukocytes were dispersed using a phosphate buffer saline (PBS)-filled syringe equipped with a 23-G needle. Residual red blood cells were resuspended in ammoniumchloride $(\mathrm{ACK})$ lysis buffer (Tris- $\mathrm{NH}_{4} \mathrm{Cl}$ ). The intact leukocytes were recovered by centrifugation at $500 \times g$ for $5 \mathrm{~min}$. After washing twice in sterile PBS, cell viability was determined by the exclusion of Trypan Blue (BD Biosciences). Viability exceeded $95 \%$ of all cell populations in all cases. Furthermore, the antigen presenting ability, NK cytotoxicity, phagocytosis of mononuclear, lymphocyte proliferation and antibody production were analyzed by fluorescence-activated cell sorting (FACS) flow cytometery (FACS calibrator; BD Biosciences).

\subsection{Immunocytostaining and flow cytometry}

Isolated cells were washing in sterile PBS and recovered by centrifugation at $500 \times \mathrm{g}$ for $5 \mathrm{~min}$. Add $100 \mu \mathrm{L}$ of $5 \mu \mathrm{g} / \mathrm{mL}$ rat antimouse CD16/CD32 antibody (2.4G , Mouse Fc Block ${ }^{\mathrm{TM}}$; BD Biosciences) in PBS for $30 \mathrm{~min}$ on ice to reduce nonspecific antibody binding by Fc receptors. Cells were then incubated with $100 \mu \mathrm{L}$ of $5 \%$ colorconjugated monoclonal antibody and primary monoclonal antibody, followed by immunoglobulin G (IgG)-fluorochrome isothiocyanate (FITC) at $4{ }^{\circ} \mathrm{C}$ for $30 \mathrm{~min}$. Three-color immunolabeling was performed using FITC-, phycoerythrin (PE)-, allophycocyanine (APC)-, and Cychrome (Cy)-conjugated monoclonal antibody and appropriate isotype controls (Serotec, San Diego, CA, USA). The immunolabeling was used for characterization of DCs, macrophage and NK cells as follows: anti-CD11c-FITC, anti-CD49b/Pan-NK-FITC, anti-I-A/I-E (MHC II)-PE, anti-NKG2D (natural killer group-2 D)-PE, anti-mouse CD80-APC, anti-CD11b-Cy5 and anti-CD86-Cy5. During all work, cells were kept at $4{ }^{\circ} \mathrm{C}$ and at low light exposure. After washing three times with PBS, cells were applied to a FACS flow cytometer and the data were collected and analyzed using CellQuest Software (BD Biosciences). The analysis was based on counting 20,000 cells.

\subsection{Preparation of crude L. paracasei subsp. paracasei NTU 101 antigen}

L. paracasei subsp. paracasei NTU 101 was cultured with MRS broth at $37^{\circ} \mathrm{C}$ for $48 \mathrm{~h}$. The cultures were centrifuged at $5,000 \times \mathrm{g}$ for $10 \mathrm{~min}$ at $4{ }^{\circ} \mathrm{C}$, and the pellet was lysed in a lysis buffer [ $350 \mathrm{mM}$ Tris- $\mathrm{HCl}$, $10 \mathrm{mM}$ EDTA (ethylene diamine tetraacetate; Sigma-Aldrich, St. Louis, MO, USA), and 1 mM PMSF (phenylmethanesulfonyl fluoride; SigmaAldrich)]. This solution was then sonicated on ice four times for 3 min (30\% cycle, model 250; Branson Ultrasonics, Danbury, CT, USA) and centrifuged for $10 \mathrm{~min}$ at $12,000 \times g$ at $4{ }^{\circ} \mathrm{C}$. The protein concentration was determined by the Bradford assay (BioRad, Munich, Germany) using bovine serum albumin (Sigma-Aldrich) as a standard, and the crude antigen was stored at $-20{ }^{\circ} \mathrm{C}$ until further use.

\subsection{Lymphocyte proliferation analysis}

Bromodeoxyuridine (BrdU) incorporation was used to measure cell growth and DNA synthesis. Splenocytes $\left(2 \times 10^{6}\right.$ cells $\left./ \mathrm{mL}\right)$ exposed or not exposed to $10 \mu \mathrm{g} \mathrm{mL} \mathrm{m}^{-1}$ or $100 \mu \mathrm{g} \mathrm{mL}^{-1}$ L. paracasei subsp. paracasei NTU $101 \mathrm{Ag}$ were seeded in 24-well tissue culture microplates (Orange Scientific, Belgium). At $18 \mathrm{~h}$ before the end of the incubation period (2 days in $5 \% \mathrm{CO}_{2}$ in a humidified $37{ }^{\circ} \mathrm{C}$ incubator), $300 \mu \mathrm{M}$ of BrdU (Sigma-Aldrich) was added per well. After incubation, the cells were then washed twice in PBS and centrifuged at $500 \times \mathrm{g}$ for $5 \mathrm{~min}$ at $4{ }^{\circ} \mathrm{C}$. Cells were incubated in $50 \mu \mathrm{L}$ PBS containing $5 \mu \mathrm{g} / \mathrm{mL}$ FITCconjugated Anti-BrdU Ab (BD Biosciences) for $30 \mathrm{~min}$ at $4{ }^{\circ} \mathrm{C}$. After washing in PBS, cells were analyzed by flow cytometry as described above. Green fluorescence from the fluorescein FITC-antibody conjugate was considered a measure of BrdU incorporation. The results were expressed as the percentage of BrdU positive cells. The stimulation index (SI) was calculated by the following formula: $\mathrm{SI}=\%$ BrdU in experimental cultures/\% BrdU in control cultures.

\subsection{Determination of antibody production}

Blood samples were collected at different times and centrifuged to separate the sera for antibody assays. Individual serum samples were stored at $-20{ }^{\circ} \mathrm{C}$ until use. IgG concentration in serum of BALB/C mice was determined by an indirect enzyme-linked immunosorbent assay (ELISA). The diluted serum (1:200) was added to each well of 
microtiter plates in $100 \mu \mathrm{L}$ aliquots. These plates were incubated $30 \mathrm{~min}$ at $37^{\circ} \mathrm{C}$. After washing three times with PBS containing $0.05 \%$ Tween-20 (PBST), 0.5\% non-fat skim milk in PBS was added for $30 \mathrm{~min}$ at $37{ }^{\circ} \mathrm{C}$ to block unoccupied sites. After blocking, each plate was washed three times with PBST prior to the addition of $100 \mu \mathrm{L}$ a $1: 1000$ dilution of horseradish peroxidase-conjugated goat-anti-mouse IgG
(Zymed, Carlsbad, CA, USA) into each well. After incubation for 30 min at $37{ }^{\circ} \mathrm{C}$, each plate was washed with PBST four times and each well received $100 \mu \mathrm{L}$ of ABTS (2,2'-Azino-bis (3-ethylbenzthiazoline-6sulfonic acid)) peroxidase substrate solution (Zymed) for $30 \mathrm{~min}$ at $37^{\circ} \mathrm{C}$. Optical density (OD) was measured at $405 \mathrm{~nm}$ in a PowerWave X340 microplate reader (Bio-Tek, Winooski, VT, USA).
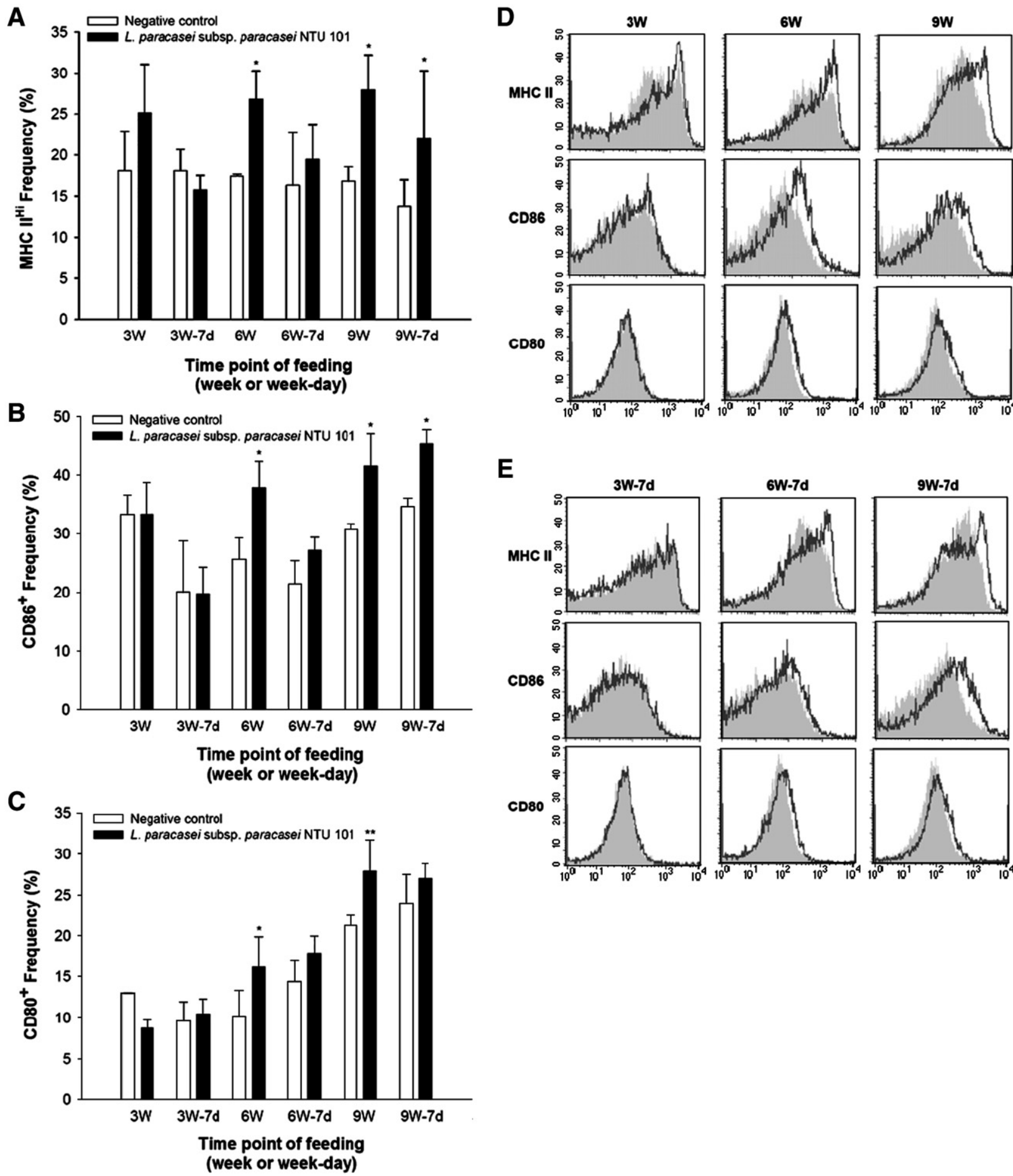

$\mathbf{E}$
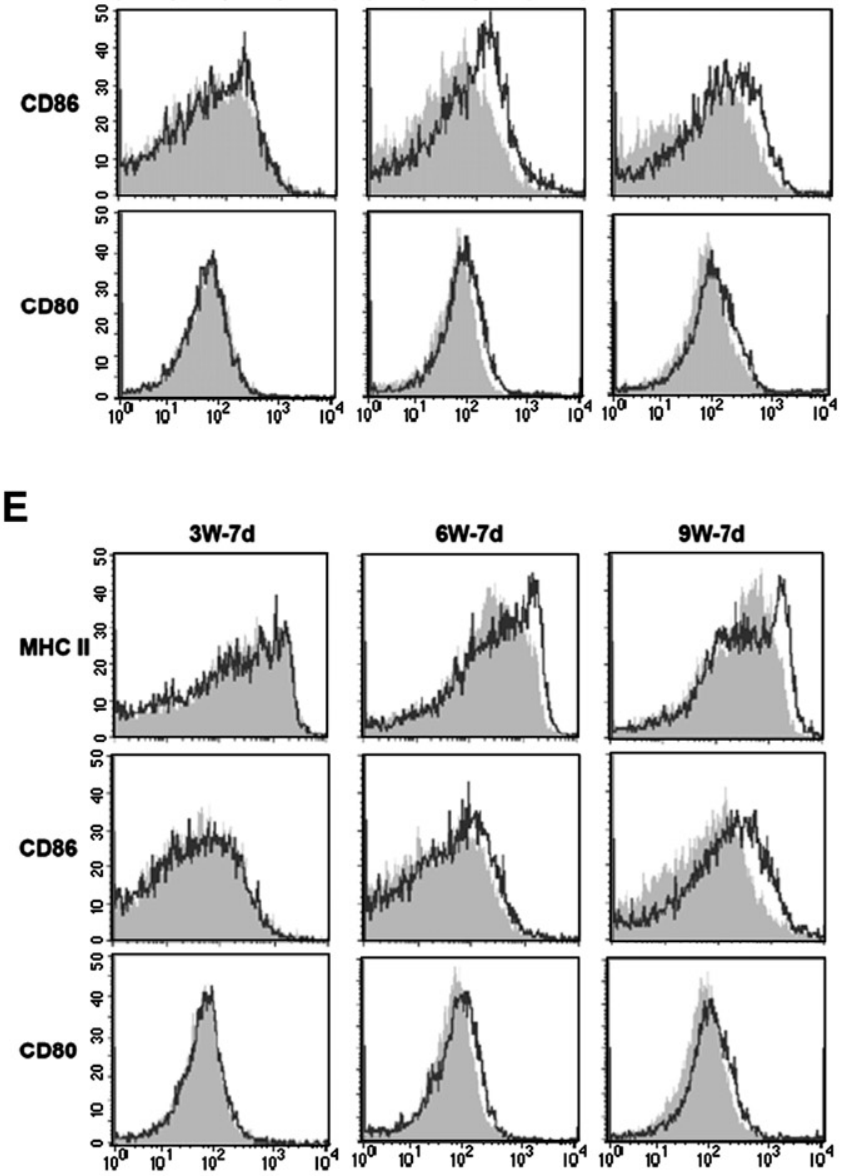

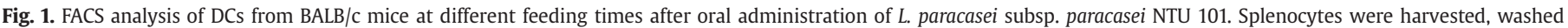

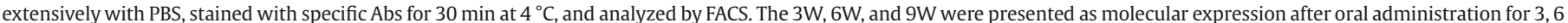

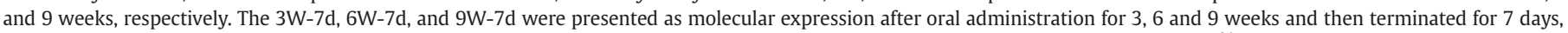

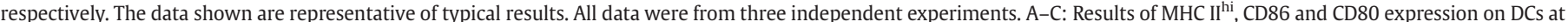

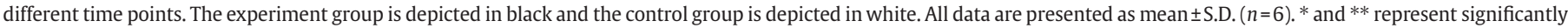

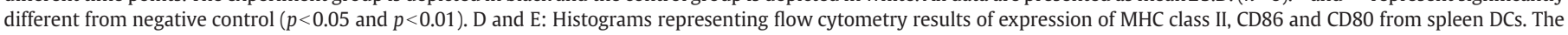
experiment group is depicted in black line and the control group is depicted in gray shade. 


\subsection{Analysis of intestinal microflora}

In the second experiment, Sprague-Dawley rats were divided into four groups. They received $10^{9} \mathrm{CFU} / \mathrm{mL}$ L paracasei subsp. paracasei NTU 101 in $0.1 \mathrm{~mL}$ MRS broth or bacteria-free MRS broth by oral administration once a day for 3, 6 and 9 consecutive weeks. All data were from three independent experiments. After the different times, the feces of each Sprague-Dawley rat was collected and placed in a capped test tube, which was taken to a lamina flow cabinet where $1 \mathrm{~g}$ of each feces sample was weighed, transferred into a tube with $9 \mathrm{~mL}$ of anaerobic diluents, and homogenized by vortexing. The same procedure was repeated several times to perform a serial dilution. The bacterial viability was determined by determining CFUs after plating on MRS (lactobacilli), BIM-25 (bifidobacteria) and TSC w/egg yolk agar (Clostridium perfringens) (BD Biosciences). The plates were placed in anaerobic containers at $37^{\circ} \mathrm{C}$ for $48 \mathrm{~h}$.

\subsection{Statistical analysis}

Values shown represent the mean $\pm S D$ of separate experiments. Data were analyzed using the one-way ANOVA procedure of SPSS software (Cary, NC, USA). The differences among means were detected by the Duncan's Multiple Range Test. Data were considered significantly different $(p<0.05)$ in variables between groups.

\section{Results}

3.1. L. paracasei subsp. paracasei NTU 101 up-regulate maturation of DC surface marker expression

To investigate surface marker expression on DCs at different time after administration with $L$. paracasei subsp. paracasei NTU 101, flow cytometry analysis was performed. Upon isolating splenocytes from mice, cells were examined by flow cytometry using the DC marker CD11c. Based on the level of MHC class II expression on the cell surface, immature and mature DC subsets can be recognized as MHC ${\text { class } \text { II }^{\text {low }} \text { and MHC class II }}^{\text {hi }}$ DC, respectively (Lutz et al., 1999). The expression of MHC was then evaluated by MHC class II ${ }^{\text {hi }}$. The data showed that MHC class II ${ }^{\text {hi }}$ were activated by L. paracasei subsp. paracasei NTU 101 at 6 and 9 weeks (Fig. 1A and D). The level of MHC class II $^{\text {hi }}$ activation was significantly different from negative control $(p<0.05)$. Interestingly, the activation of MHC class II $^{\text {hi }}$ was $112.5 \%$ greater than the negative control in $9 \mathrm{~W}-7 \mathrm{~d}$ group $(p<0.05)$ (Fig. 1E). Similar results of MHC class II molecule expression on macrophages were also observed (data not shown).

The expression of co-stimulatory molecules CD86 (B7.2) and CD80 (B7.1) activation were significantly different from that of negative control after administration for 6 weeks $(p<0.05)$ (Fig. 1B and C). In the $6 \mathrm{~W}-7 \mathrm{~d}$ group, activation of CD80 and CD86 were increased $26.5 \%$
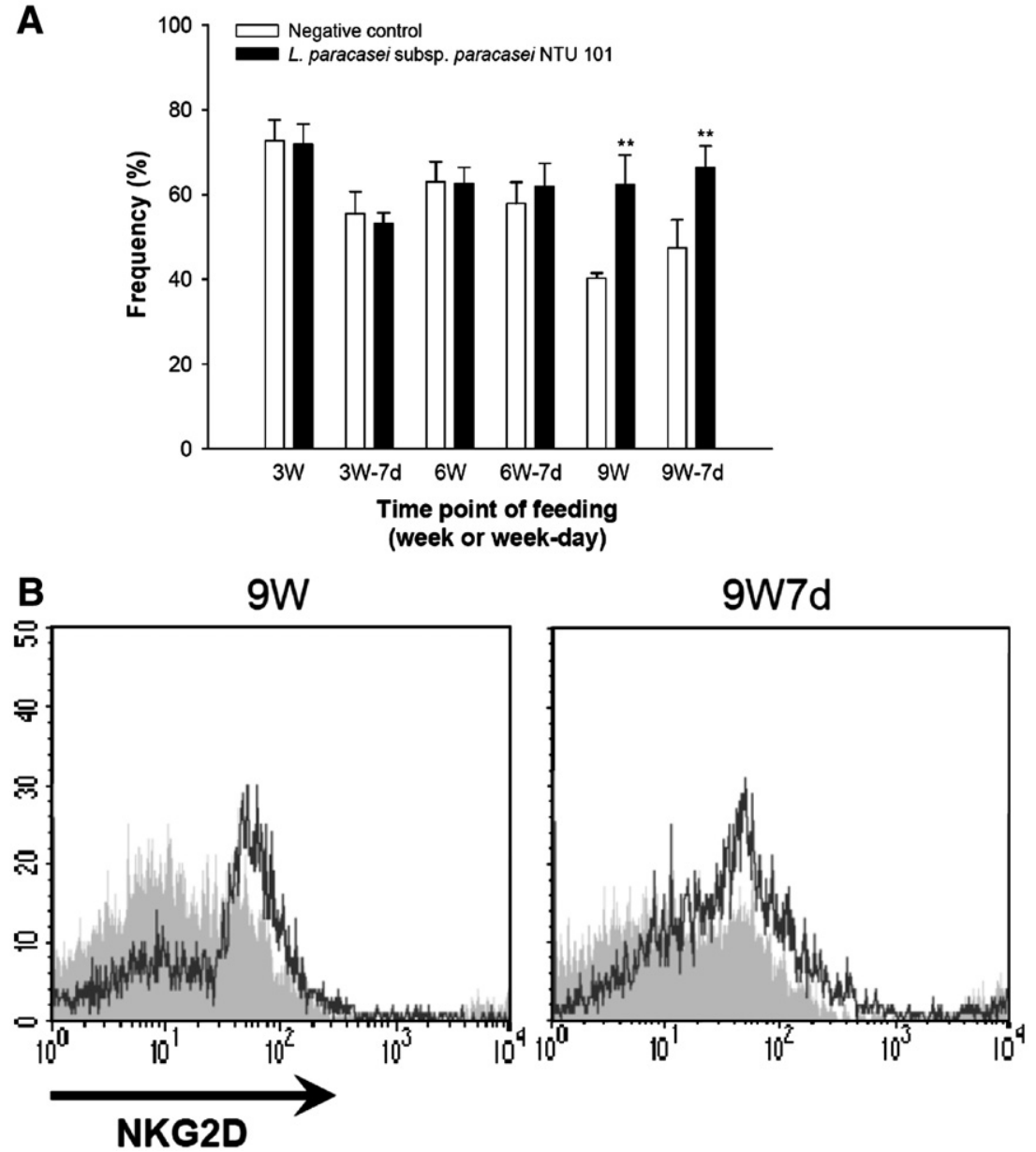

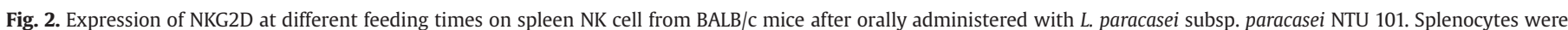

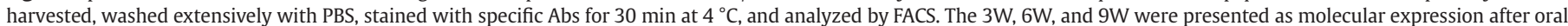

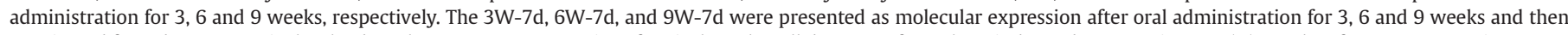

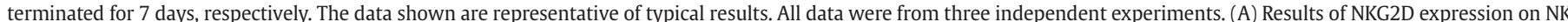

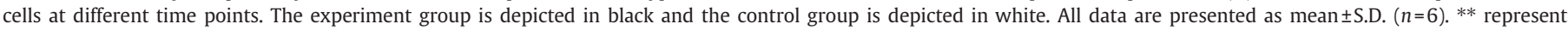

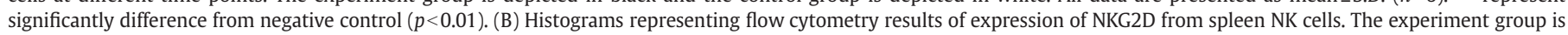
depicted in black line and the control group is depicted in gray shade. 
and 24.3\%, respectively, relative to the negative control, but only CD86 molecule activation was constantly expression even through the 7 day bacteria-free diet following the 9 week administration of lactobacilli $(p<0.05)$ (Fig. 1E). These results indicated L. paracasei subsp. paracasei NTU 101 could enhance antigen presenting ability on DCs, and that the persistence of the surface marker was time-dependent.

\subsection{Enhancement of NKG2D triggers NK cell-mediated cytotoxicity by L. paracasei subsp. paracasei NTU 101}

Previous studies have shown that NKG2D receptor can be constitutively expressed on NK cell, and that the NKG2D-DAP-10 complex can trigger cytotoxicity accompanied with killing of tumor cells following NKG2D cross-linking in NK cells (Coudert and Held, 2006). To determine whether activation of cytotoxicity via NKG2Ddependent NK cells is mediated by $L$. paracasei subsp. paracasei NTU 101 , splenocytes from mice were verified by flow cytometry using the NK cell marker NK1.1. NK cell numbers in splenocytes were not different among any of the time intervals (data not shown). As seen in Fig. 2A, NKG2D expression decreased after 9 weeks in control mice. But the level of activation was significantly increased after oral administration of $L$. paracasei subsp. paracasei NTU 101 for 9 weeks $(p<0.01)$. Moreover, the activation of NKG2D was constantly expressed in the $9 \mathrm{~W}-7 \mathrm{~d}$ group ( $p<0.01$, Fig. $2 \mathrm{~B}$ ). These observations indicated that $L$. paracasei subsp. paracasei NTU 101 could up-regulate cytotoxicity via NKG2D-dependent pathway and that the persistent expression was time-dependent.

\subsection{L. paracasei subsp. paracasei NTU 101-induced proliferation of spleen lymphocytes}

According to our previous experiments, DC activation was induced by L. paracasei subsp. paracasei NTU 101 after administration for 6 weeks. Based on the knowledge that DCs activate the adaptive immune response by priming lymphocyte to proliferate and produce antibody, the influence of Lactobacillus on lymphocyte proliferation was measured. After isolation of splenocytes, the cells were incubated with $L$. paracasei subsp. paracasei NTU $101 \mathrm{Ag}$ at different time intervals. The effect of immunostimulation was evaluated by stimulation index. As shown in Table 1, splenocytes from control mice showed spontaneous proliferation at different time intervals. However, the proliferation levels of splenocytes from treated mice were significantly higher than those of control mice after administration for 9 weeks $(p<0.05)$. Similarly, strong proliferation was evident in the $9 \mathrm{~W}-7 \mathrm{~d}$ group $(p<0.05)$, whereas no difference was found among other time intervals after feeding Lactobacillus. These results suggested that lymphocyte proliferation affected by $L$. paracasei subsp. paracasei NTU 101 was time-dependent.

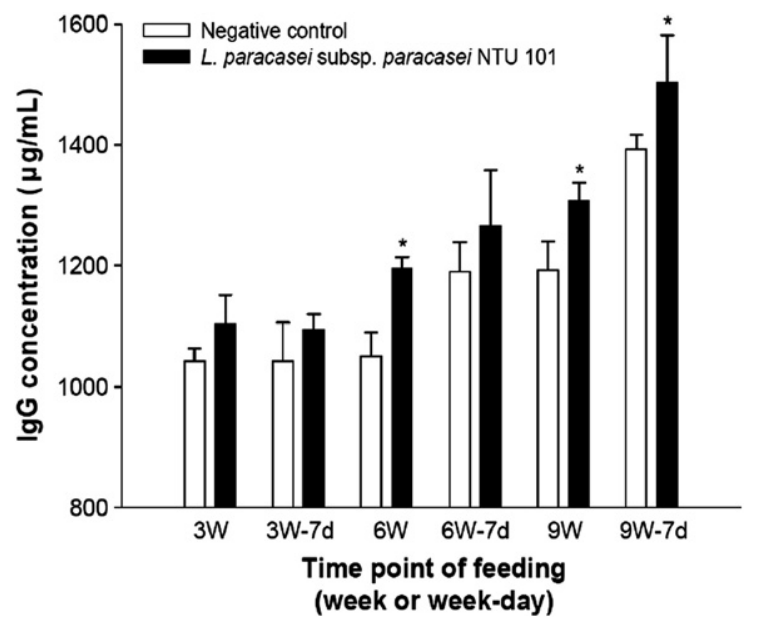

Fig. 3. Production of IgG antibodies at different feeding times from BALB/c mice after orally administered with $L$. paracasei subsp. paracasei NTU 101 . All data are presented as mean \pm S.D. $(n=6)$. The experiment group is depicted in black and the control group is depicted in white. ${ }^{*}$ represents significantly different from negative control $(p<0.05)$. The $3 \mathrm{~W}, 6 \mathrm{~W}$ and $9 \mathrm{~W}$ represent antibody concentration change after oral administration for 3,6 and 9 weeks. The $3 W-7 d, 6 W-7 d$ and $9 W-7 d$ represent antibody concentration change after oral administration for 3, 6 and 9 weeks and then termination for 7 days. All data were from three independent experiments.

\subsection{Production of serum $\operatorname{Ig} G$ antibody after L. paracasei subsp. paracasei NTU 101 administration}

Following Ag recognition by Ag-presenting cells, B cells become activated and differentiated into antibody-secreting plasma cells to produce IgG antibodies, a most important antibody of adaptive immunity (Ollila and Vihinen, 2005). Therefore, we examined the effects of Lactobacillus on the IgG level in mice serum by sandwich ELISA. The data showed that after feeding mice with $L$. paracasei subsp. paracasei NTU 101 for 6 or 9 weeks significantly increased IgG antibodies in serum compared with control mice $(p<0.05)$. The increased concentration of IgG antibodies was maintained in the $9 \mathrm{~W}$ $7 d$ group ( $p<0.05$, Fig. 3 ), but not in the $3 \mathrm{~W}-7 \mathrm{~d}$ and $6 \mathrm{~W}-7 \mathrm{~d}$ groups, indicative of the time-dependence of the effect.

\subsection{L. paracasei subsp. paracasei NTU 101 regulation of microflora in the intestinal tract}

In previews experiments, the expression of innate and adaptive immunity in mice was enhanced by $L$. paracasei subsp. paracasei NTU 101 and the activation of immune cells was time-dependent. To better understand the possible mechanism of the enhanced immune response, we sought to determine whether intestinal microflora

Table 1

Effect of L. paracasei subsp. paracasei NTU 101 administration to mice on slpeen lymphocyte proliferation under stimulation with homologous antigen preparation

\begin{tabular}{|c|c|c|c|c|c|c|c|c|c|c|}
\hline \multirow[t]{3}{*}{ Feeding time $^{\mathrm{a}}$} & \multicolumn{5}{|l|}{ Control } & \multicolumn{5}{|l|}{ Experiment } \\
\hline & \multirow[t]{2}{*}{ Non-treated ${ }^{b}$} & \multicolumn{2}{|l|}{$1 \times$ NTU101 } & \multicolumn{2}{|l|}{$10 \times$ NTU101 } & \multirow[t]{2}{*}{ Non-treated } & \multicolumn{2}{|l|}{$1 \times$ NTU101 } & \multicolumn{2}{|l|}{$10 \times$ NTU101 } \\
\hline & & & $\mathrm{SI}^{\mathrm{C}}$ & & SI & & & SI & & SI \\
\hline $3 W$ & $4.52 \pm 0.26$ & $5.31 \pm 0.18$ & 1.18 & $5.67 \pm 0.49$ & 1.25 & $4.62 \pm 0.40$ & $5.56 \pm 0.37$ & 1.20 & $6.25 \pm 0.41$ & 1.35 \\
\hline $6 \mathrm{~W}$ & $5.41 \pm 1.42$ & $6.70 \pm 1.05$ & 1.24 & $10.29 \pm 1.31$ & 1.90 & $6.03 \pm 0.77$ & $8.74 \pm 0.56$ & 1.45 & $13.74 \pm 2.51$ & 2.28 \\
\hline $9 \mathrm{~W}$ & $6.45 \pm 0.35$ & $11.38 \pm 1.01$ & 1.76 & $18.32 \pm 3.60$ & 2.84 & $7.77 \pm 0.97$ & $20.46 \pm 0.59^{d}$ & 2.63 & $29.85 \pm 3.33^{d}$ & 3.84 \\
\hline $3 W-7 d$ & $4.81 \pm 0.41$ & $5.96 \pm 0.35$ & 1.24 & $6.01 \pm 0.49$ & 1.25 & $5.07 \pm 0.24$ & $6.78 \pm 0.41$ & 1.34 & $6.96 \pm 0.22$ & 1.37 \\
\hline $6 W-7 d$ & $6.52 \pm 0.41$ & $7.97 \pm 0.66$ & 1.22 & $10.52 \pm 1.92$ & 1.61 & $7.05 \pm 0.24$ & $8.77 \pm 0.37$ & 1.24 & $12.39 \pm 1.47$ & 1.76 \\
\hline $9 W-7 d$ & $6.62 \pm 0.14$ & $8.21 \pm 0.98$ & 1.24 & $17.22 \pm 1.08$ & 2.60 & $6.86 \pm 0.43$ & $11.49 \pm 2.17^{d}$ & 1.67 & $24.03 \pm 2.91^{d}$ & 3.50 \\
\hline
\end{tabular}

All data from three independent experiments are presented as mean \pm S.D. $(n=6)$.

The control group was fed with MRS broth only, and the experiment group was fed with $10^{8}$ CFU of $L$. paracasei subsp. paracasei NTU $101 /$ daily for different times.

a The $3 \mathrm{~W}, 6 \mathrm{~W}$ and $9 \mathrm{~W}$ represent oral administration for 3,6 , and 9 weeks. The $3 \mathrm{~W}-7 \mathrm{~d}, 6 \mathrm{~W}-7 \mathrm{~d}$, and $9 \mathrm{~W}-7 \mathrm{~d}$ were presented after oral administration for 3, 6, and 9 weeks and then termination for 7 days.

b FACS analysis after stimulation without (non-treated) or with $10 \mu \mathrm{g} \mathrm{mL}{ }^{-1}$ (1×) and $100 \mu \mathrm{gL} \mathrm{m}^{-1}$ (10×) NTU 101 antigen for 2 days.

c SI was calculated by the following formula: $\mathrm{SI}=\%$ BrdU in experimental cultures $/ \%$ BrdU in control cultures.

d Significant difference $(p<0.05)$ from the cells of control mice with the same dose of antigen stimulation. 
Table 2

Quantitative analysis of intestinal microflora at different times after administration of $L$. paracasei subsp. paracasei NTU 101

\begin{tabular}{llllll}
\hline & Group & \multicolumn{4}{l}{ Feeding time $\left(\log _{10} \mathrm{CFU} / \mathrm{g}\right)$} \\
\cline { 3 - 6 } & & 0 & 3 & 6 & 9 \\
\hline Bifidobacterium spp & Control & $6.75 \pm 0.61$ & $6.75 \pm 0.39$ & $6.37 \pm 0.26$ & $6.87 \pm 0.32$ \\
& Experiment & $6.86 \pm 0.34$ & $7.11 \pm 0.54$ & $6.86 \pm 0.49^{*}$ & $7.35 \pm 0.62^{*}$ \\
Lactobacillus spp & Control & $7.47 \pm 0.42$ & $7.63 \pm 0.18$ & $7.32 \pm 0.40$ & $7.49 \pm 0.17$ \\
& Experiment & $7.35 \pm 0.63$ & $7.73 \pm 0.22$ & $7.19 \pm 0.26$ & $7.85 \pm 0.27^{*}$ \\
C. perfringens & Control & $3.19 \pm 0.41$ & $3.22 \pm 0.56$ & $3.98 \pm 0.52$ & $2.66 \pm 0.61$ \\
& Experiment & $2.98 \pm 0.88$ & $3.21 \pm 0.54$ & $3.24 \pm 0.50^{*}$ & $2.15 \pm 0.47^{*}$ \\
\hline
\end{tabular}

All data from three independent experiments are presented as mean \pm S.D. $(n=8)$. The control group was fed with MRS broth only, and the experiment group was fed with $10^{8}$ CFU of $L$. paracasei subsp. paracasei NTU 101/daily for different times.

$*$ Significantly different from control group $(p<0.05)$.

were involved. We determined the bacterial counts in the feces at different time intervals. As shown in Table 2, bifidobacteria and lactobacilli counts were significantly increased after feeding for 6 or 9 weeks, respectively $(p<0.05)$. Furthermore, $C$. perfringens was markedly decreased after feeding for 6 and 9 weeks compared with control mice $(p<0.05)$.

\section{Discussion}

Many Lactobacillus species regulate the innate and adaptive immune responses, including modulated expression of cytokines, maturation of surface markers in immune cells, lymphocyte responses or antibody production (He et al., 2005). Lactobacilli are potent inducers of Th1-type cytokines such as IL-2, IFN- $\gamma$, and TNF- $\alpha$ in a dose-dependent manner, but not of Th2 cytokines (Hessle et al., 1999). Although previous studies have established that lactobacilli are able to enhance immunity in mice, and this effect is dose- and straindependent (Paturi et al., 2007), correlation between administration time and regulation of immunity by Lactobacillus remains unclear. Therefore, the interaction of $L$. paracasei subsp. paracasei NTU 101 and immunity was investigated in the present study.

Initiation of the immune response requires activation of MHC class II and co-stimulatory signals in the form of CD80 and CD86 molecules provided by professional antigen presenting cells (APC) (Harris and Ronchese, 1999). Previous study has established that DC maturation is up-regulated though the expression of C80, CD86, and MHC class II after interaction with probiotics. Moreover, the regulation of DC cytokines by probiotics may contribute to the benefit in treating the intestinal diseases (Drakes et al., 2004). Therefore, DCs play a crucial immunoregulatory role in immune responses under antigen uptake and processing such as the induction of Ag-specific immune responses and Th1/Th2 balance. Different Lactobacillus species cause distinct expression of MHC class II, CD80 and CD86 molecules on DCs (Christensen et al., 2002). In this study, we also observed upregulation after administration with $L$. paracasei subsp. paracasei NTU 101 in 6W, 9W and 9W-7d groups. MHC class II activity on macrophage also showed a similar result. The results suggest Lactobacillus not only enhances APC activation in the feeding period, but also the persistence of activation under long-period treatment. DC maturation not only induces the adaptive immune response, but also supports the tumoricidal activity or early stages of viral infection on NK cells (Fernandez et al., 1999). Furthermore, Lactobacillus significant increases cell populations and cytotoxicity ability on NK cells in mice and human (Lee et al., 2004; Takeda and Okumura, 2007). NKG2D is one of the major activating receptors on NK cells, which mediate cytotoxic activity by recognizing defined ligands, often over-expressed on transformed cells (Cerwenka and Lanier, 2001). The abolition of tumor cell killing when NKG2D-blocking antibody is added suggests that cytotoxicity depends on NKG2D receptor function (Ostberg et al.,
2007). Here, we used NKG2D to evaluated cytotoxic activity. We observed that NKG2D decreased with time, whereas expression still continue to be enhanced after administration with $L$. paracasei subsp. paracasei NTU 101 in the elderly mice. These data demonstrate that innate immunity, evident as APC and NK cell activity, are increased by L. paracasei subsp. paracasei NTU 101 in a time-dependent manner.

The cells of the adaptive immune system are lymphocytes. $\mathrm{T}$ and $\mathrm{B}$ cells are the major types of lymphocytes. T cells are immune response mediators, and play an important role in establishing and maximizing the capabilities of the adaptive immune response. B cells are the major cells involved in the creation of antibodies that circulate in blood plasma and lymph. Activation by APC interaction in turn spurs activation of naïve lymphocyte; their subsequent activation, proliferation and differentiation leads to the secretion of antibodies and cytokines. Previously, it was demonstrated that oral administration of Lactobacillus enhances specific adaptive immune responses via innate immunity in mice (Galdeano and Perdigon, 2006). In the present study, spleen cells and serum from mice fed with $L$. paracasei subsp. paracasei NTU 101 showed significant increases of lymphocytes and antibody production, and the expression pattern was similar to our previous DCs maturation experiment. Based on the above findings, we suggest that activation of adaptive immunity is regulated by DC activation. Furthermore, L. paracasei subsp. paracasei NTU 101 not only enhances the immunity in the feeding period, but also the persistence of activation under long-period treatment.

Probiotics beneficially affect the host by improving the balance of the intestinal flora (Fuller, 1989). Probiotic intake should lead to the creation of gut microbiology conditions that suppress harmful microorganisms (Rada and Rychly, 1995; Mountzouris et al., 2007) and favor beneficial microorganisms, ultimately enhancing gut health. In a previous study, we demonstrated the number of bifidobacteria and lactobacilli are increased in the cecum of hypercholesterolemic hamsters after feeding with $L$. paracasei subsp. paracasei NTU 101 (Chiu et al., 2006). To better understand the effect of intestinal microflora and immunity, we measured probiotics and harmful microorganisms in the intestinal tract at different time intervals. The results showing the altered composition of intestinal microflora support the suggestion that the persistence of immune activation may be caused by the intestinal probiotics.

In conclusion, $L$. paracasei subsp. paracasei NTU 101 is able to persistently enhance both innate and adaptive immune responses, e.g DC maturation, NK cell activity, lymphocyte proliferation and antibody production. It is noteworthy that the ability to stimulate immune response is time-dependent. Furthermore, the persistence of enhancing immunity may be due to the regulation of intestinal microflora, increased probiotics and suppression of harmful microorganisms. This paper, which represents the first report of the enhancement of immunity by Lactobacillus in time-dependent manner, raises an interesting possibility that supplementation of human diets with L. paracasei subsp. paracasei NTU 101 may convey a health benefit.

\section{References}

Aderem, A., Underhill, D.M., 1999. Mechanisms of phagocytosis in macrophages. Annua Reviews of Immunology 17, 593-623.

Ahrne, S., Nobaek, S., Jeppsson, B., Adlerberth, I., Wold, A.E., Molin, G., 1998. The normal Lactobacillus flora of healthy human rectal and oral mucosa. Journal of Appled Microbiology 85, 88-94.

Cerwenka, A., Lanier, L.L., 2001. Natural killer cells, viruses and cancer. Nature Review Immunology 1, 41-49.

Chiu, C.H., Lu, T.Y., Tseng, Y.Y., Pan, T.M., 2006. The effects of Lactobacillus-fermented milk on lipid metabolism in hamsters fed on high-cholesterol diet. Applied Microbiology and Biotechnology 71, 238-245.

Christensen, H.R., Frokiaer, H., Pestka, J.J., 2002. Lactobacilli differentially modulate expression of cytokines and maturation surface markers in murine dendritic cells. Journal of Immunology 168, 171-178.

Cotter, P.D., Hill, C., Ross, R.P., 2005. Bacteriocins: developing innate immunity for food Nature Reviews Microbiology 3, 777-788.

Coudert, J.D., Held, W., 2006. The role of the NKG2D receptor for tumor immunity. Seminars in Cancer Biology 16, 333-343. 
Diefenbach, A., Jamieson, A.M., Liu, S.D., Shastri, N., Raulet, D.H., 2000. Ligands for the murine NKG2D receptor: expression by tumor cells and activation of NK cells and macrophages. Nature Immunology 1, 119-126.

Drakes, M., Blanchard, T., Czinn, S., 2004. Bacterial probiotic modulation of dendritic cells. Infection and Immunity 72, 3299-3309.

Fernandez, N.C., Lozier, A., Flament, C., Ricciardi-Castagnoli, P., Bellet, D., Suter, M., Perricaudet, M., Tursz, T., Maraskovsky, E., Zitvogel, L., 1999. Dendritic cells directly trigger NK cell functions: cross-talk relevant in innate anti-tumor immune responses in vivo. Nature Medicine 5, 405-411.

Fuller, R., 1989. Probiotics in man and animals. Journal of Applied Bacteriology 66, 365-378.

Galdeano, C.M., Perdigon, G., 2006. The probiotic bacterium Lactobacillus casei induces activation of the gut mucosal immune system through innate immunity. Clinical Vaccine Immunology 13, 219-226.

Gill, H.S. Rutherfurd, K.J. Prasad, J. Gopal, P.K., 2000. Enhancement of natural and acquired immunity by Lactobacillus rhamnosus (HN001), Lactobacillus acidophilus (HN017) and Bifidobacterium lactis (HN019). British Journal of Nutrition 83, 167-176.

Goldin, B.R., 1998. Health benefits of probiotics. British Journal of Nutrition 80 S203-S207.

Harris, N.L., Ronchese, F., 1999. The role of B7 costimulation in T-cell immunity Immunology Cell Biology 77, 304-311.

He, F., Morita, H., Kubota, A., Ouwehand, A.C., Hosoda, M., Hiramatsu, M., Kurisaki, J., 2005. Effect of orally administered non-viable Lactobacillus cells on murine humoral immune responses. Microbiology and Immunology 49, 993-997.

Hessle, C., Hanson, L.A., Wold, A.E., 1999. Lactobacilli from human gastrointestinal mucosa are strong stimulators of IL-12 production. Clinical and Experimental Immunology 116, 276-282.

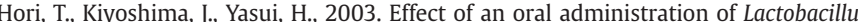
casei strain Shirota on the natural killer activity of blood mononuclear cells in aged mice. Bioscience and Biotechnology Biochemistry 67, 420-422.

Lee, J.W., Shin, J.G., Kim, E.H., Kang, H.E., Yim, I.B., Kim, J.Y., Joo, H.G., Woo, H.J., 2004. Immunomodulatory and antitumor effects in vivo by the cytoplasmic fraction of Lactobacillus casei and Bifidobacterium longum. Journal of Veterinary Science 5 , 41-48.

Lin, F.M., Chiu, C.H., Pan, T.M., 2004. Fermentation of milk-soymilk and Lycium chinense Miller mixture using a new isolate of Lactobacillus paracasei subsp. paracasei NTU101 and Bifidobacterium longum. Journal of Industrial Microbiology and Biotechnology 31, 559-564.

Lutz, M.B., Kukutsch, N., Ogilvie, A.L., Rossner, S., Koch, F., Romani, N., Schuler, G., 1999. An advanced culture method for generating large quantities of highly pure dendritic cells from mouse bone marrow. Journal of Immunological Methods 223 77-92.
Maassen, C.B., van Holten-Neelen, C., Balk, F., den Bak-Glashouwer, M.J., Leer, R.J., Laman, J.D., Boersma, W.J., Claassen, E., 2000. Strain-dependent induction of cytokine profiles in the gut by orally administered Lactobacillus strains. Vaccine 18, 2613-2623.

Meydani, S.N., Ha, W.K., 2000. Immunologic effects of yogurt. American Journal of Clinical Nutrition 71, 861-872.

Mohamadzadeh, M., Olson, S., Kalina, W.V., Ruthel, G., Demmin, G.L., Warfield, K.L., Bavari, S., Klaenhammer, T.R., 2005. Lactobacilli activate human dendritic cells that skew T cells toward T helper 1 polarization. Proceedings of the National Academy of Science USA 102, 2880-2885.

Morrissette, N., Gold, E., Aderem, A., 1999. The macrophage-a cell for all seasons. Trends in Cell Biology 9, 199-201.

Mountzouris, K.C., Tsirtsikos, P., Kalamara, E., Nitsch, S., Schatzmayr, G., Fegeros, K., 2007. Evaluation of the efficacy of a probiotic containing Lactobacillus, Bifidobacterium, Enterococcus, and Pediococcus strains in promoting broiler performance and modulating cecal microflora composition and metabolic activities. Poultry Science 86, 309-317.

Ollila, J., Vihinen, M., 2005. B cells. International Journal of Biochemistry and Cell Biology 37, 518-523.

Ostberg, J.R., Dayanc, B.E., Yuan, M., Oflazoglu, E., Repasky, E.A., 2007. Enhancement of natural killer (NK) cell cytotoxicity by fever-range thermal stress is dependent on NKG2D function and is associated with plasma membrane NKG2D clustering and increased expression of MICA on target cells. Journal of Leukocyte Biology 82, $1322-1331$.

Pan, T.M., Chiu, C.H., Guu, Y.K., 2002. Characterization of Lactobacillus isolates from pickled vegetables for use as dietary or pickle adjuncts. Foods and Food Ingredients Journal of Japan 206, 45-51.

Paturi, G., Phillips, M., Jones, M., Kailasapathy, K., 2007. Immune enhancing effects of Lactobacillus acidophilus LAFTI L10 and Lactobacillus paracasei LAFTI L26 in mice. International Journal of Food Microbiology 115, 115-118.

Perdigon, G., Vintini, E., Alvarez, S., Medina, M., Medici, M., 1999. Study of the possible mechanisms involved in the mucosal immune system activation by lactic acid bacteria. Journal of Dairy Science 82, 1108-1114.

Rada, V., Rychly, I., 1995. The effect of Lactobacillus salivarius administration on coliform bacteria and enterococci in the crop and cecum of broiler chickens. Veterinary Medicine (Praha) 40, 311-315.

Takeda, K., Okumura, K., 2007. Effects of a fermented milk drink containing Lactobacillus casei strain Shirota on the human NK-cell activity. Journal of Nutrition 137, 791S-793S 